\title{
NÍVEIS CRÍTICOS DE FÓSFORO, PARA MILHO, EM CASA DE VEGETAÇÃO, DE ACORDO COM A SUA LOCALIZAÇÃO NO SOLO ${ }^{(1)}$
}

\author{
Paulo Batista Leite ${ }^{(2)}$, Víctor Hugo Alvarez V. ${ }^{(3)}$, Nairam Félix de \\ $\operatorname{Barros}^{(3)}$, Júlio César Lima Neves ${ }^{(3)} \&$ André Guarçoni M. ${ }^{(4)}$
}

\begin{abstract}
RESUMO
A aplicação localizada de $P$ no solo apresenta grande influência sobre sua disponibilidade, pois diminui as reações de adsorção, precipitação e fixação, em virtude da redução da superfície de contato com o solo. Em casa de vegetação, estudou-se a calibração de $\mathbf{P}$ diante da diferença de teores no solo provocada por sua localização. Foram usadas amostras de um LVAd de Viçosa e de um LVAm de Lassance. Utilizaram-se 14 combinações de sete doses de $\mathrm{P}$ adicionadas na parte central e superior do vaso ("cova") com sete doses de $\mathbf{P}$ adicionadas no solo circundante à "cova" (solo "externo"). As amostras dos dois locais foram incubadas por $20 \mathrm{~d}$, com calcário, e por $40 \mathrm{~d}$, com $\mathrm{P}$, em embalagens separadas e depois acondicionadas no vaso. As sementes de milho foram semeadas no meio da "cova". As plantas foram cultivadas por $32 \mathrm{~d}$. Determinaram-se a produção de matéria seca da parte aérea, o teor de $\mathbf{P}$ por Mehlich-1 nos dois locais do solo em amostras colhidas depois da incubação e antes do plantio e de uma amostra composta pelo solo da "cova" mais o "externo" da parte superior, após a colheita. A produção de matéria seca não se relacionou bem com a quantidade de $P$ aplicada por vaso, mas, sim, com as doses aplicadas nos dois locais, mostrando o efeito da localização. $O$ crescimento da planta dependeu essencialmente da dose aplicada na "cova". Os elevados valores de nível crítico para a "cova" não implicam o uso de grande quantidade de $\mathbf{P}$ por vaso. $\mathrm{O}$ teor de $\mathrm{P}$ na amostra da metade superior do solo do vaso retirada após a colheita representou bem a disponibilidade média, porém não se relacionou bem com o crescimento da planta, o que dificulta a obtenção de um nível crítico para amostras que representem a fertilidade média para todo o volume de solo. Sugerem-se mudanças no método de calibração de $\mathrm{P}$, em condições de aplicação localizada do $P$.
\end{abstract}

Termos de indexação: amostragem, P disponível, calibração, doses recomendáveis.

\footnotetext{
(1) Parte da Tese de Mestrado do primeiro autor, apresentada ao curso de Solos e Nutrição de Plantas da Universidade Federal de Viçosa - UFV. Recebido para publicação em janeiro de 2003 e aprovado em abril de 2006.

(2) Engenheiro-Agrônomo, M.Sc. Pioneer Sementes Ltda. CEP 75500-000 Itumbiara (GO). E-mail: paulo.leite@pioneer.com

(3) Professor do Departamento de Solos, Universidade Federal de Viçosa - UFV. CEP 36571-000 Viçosa (MG). Bolsista do CNPq. E-mail:vhav@ufv.br; nfbarros@ufv.br; julio_n2003@yahoo.com.br

(4) Pesquisador da INCAPER. Linhares (ES). E-mail: agmfertsolo@hotmail.com
} 


\title{
SUMMARY: CRITICAL PHOSPHORUS LEVELS FOR GREENHOUSE-GROWN CORN, AS A FUNCTION OF ITS PLACEMENT IN THE SOIL
}

\begin{abstract}
Localized application of $P$ in the soil has great influence on its availability because it reduces de adsorption reactions, precipitation and fixation, due to the lowered soil contact surface area. This study, carried out in a greenhouse, aimed to calibrate P soil concentrations for corn growth as affected by fertilizer placement. Samples of two Oxisols (LVAd and LVAm) were used. The treatment consisted of 14 combinations of 7 P rates, which were placed in holes in the upper mid part of the pot, with 7 Pdoses applied in the soil around the holes. Soil samples from both localizations were incubated separately with limestone for 20 days and with P for 40 days before being placed in the pots. Corn seeds were sown in the same holes as the fertilizer. The corn plants were grown for 32 days and the shoot dry matter determined. Soil samples were collected from both positions of fertilizer placement before sowing and after harvesting the plant, plus a composite sample of both soil positions after harvest. The soil P content was determined by Mehlich-1. Dry matter yield was not closely related with the applied P per pot, but it was related with the doses applied in both positions, which demonstrated the effect of localization. Plant growth depended essentially on the dose applied in the "hole". The high critical Plevel determined for this placement does not imply the use of a high amount of P per pot. The P content determined in the upper soil part after the harvest represented well the mean P availability, but did not show a close relationship with plant growth, indicating the difficulty of establishing a critical level that would represent the mean fertility of the whole soil volume. We suggest adjustments in the calibration method for P that would take into account the localized P applications.
\end{abstract}

Index terms: sampling, available $P$, calibration, recommended doses.

\section{INTRODUÇÃO}

A forma de localizar fertilizantes fosfatados solúveis em parte do volume de solo cultivado, no sentido de otimizar o uso de $\mathrm{P}$ pelas plantas, é prática recomendável para a exploração da maioria dos solos brasileiros, deficientes e com elevado poder tampão para este nutriente.

A aplicação do $\mathrm{P}$ em fração do volume de solo tem a vantagem de proteger o $\mathrm{P}$ contra as reações de adsorção, precipitação e de fixação, visto que diminui o contato deste nutriente com o solo. Com isso, na fração fertilizada, haverá alto teor do nutriente em forma disponível (Model \& Anghinoni, 1992), enquanto, na fração restante, esse permanecerá inalterado, uma vez que o P é praticamente imóvel no solo (Bray, 1954). Com a aplicação da fonte de fósforo no sulco de plantio ou na cova, a planta explora, inicialmente, uma região do solo com alta disponibilidade do nutriente e, à medida que cresce, passa a explorar um volume de solo com menor disponibilidade, o que, para Zang \& Barber (1992), pode restringir o crescimento de raízes e da parte aérea das plantas de milho. Na mesma condição, Anghinoni (1992) observou elevado crescimento inicial das plantas de milho, mas não em rendimento de grãos.

Nos trabalhos de calibração de métodos de análise de solos, define-se o nível crítico como a concentração do nutriente no solo que corresponde à disponibilidade necessária para atingir a produção de máxima eficiência econômica (Alvarez V., 1996). Todavia, em condições de localização do $\mathrm{P}$ em menor volume de solo, torna-se difícil definir a disponibilidade do nutriente para as plantas pelo método tradicional, com definição de um valor de nível crítico indiferente à localização do fertilizante, já que, com a localização, a resposta da planta passará a ser função das diferentes concentrações de P no volume de solo explorado pelas raízes. Os trabalhos de calibração do $\mathrm{P}$ devem considerar as condições de aplicação dos fertilizantes e a forma de amostragem dos solos para as quais as recomendações serão geradas, ou seja, a forma como serão utilizados pelos agricultores.

A relação entre as quantidades de $\mathrm{P}$ recuperadas do solo com as doses adicionadas varia com a localização do adubo e, conseqüentemente, com a forma de amostragem (Vasconcelos et al., 1982, Alvarez V., 1996 e Guarçoni M., 2001). Em conseqüência , também devem variar os valores dos níveis críticos. Assim, a forma de amostragem do solo para análise, quando se faz a aplicação localizada do $\mathrm{P}$, torna-se muito importante, uma vez que o resultado da análise não deve expressar apenas a disponibilidade do nutriente representativa do volume de solo, mas também aquela que se relacione com a resposta da cultura.

Este trabalho objetivou estudar a calibração de $\mathrm{P}$ disponível, determinado por Mehlich-1, para o crescimento de plantas de milho, diante da diferença de teores no solo provocada por sua localização.

\section{MATERIAL E MÉTODOS}

No experimento, realizado em casa de vegetação, foi utilizada amostra de um Latossolo Vermelho- 
Amarelo distrófico, coletada na camada de $20-50 \mathrm{~cm}$ de profundidade, em Viçosa-MG (Solo LVAd-VI), e de um Latossolo Vermelho-Amarelo textura média distrófico, coletada nos $20 \mathrm{~cm}$ superficiais, em Lassance-MG (Solo LVAm-LS) (Quadro 1).

Os tratamentos derivaram da combinação de sete doses de $\mathrm{P}$ aplicadas em $0,53 \mathrm{dm}^{3}$ de solo, equivalentes a $10 \%$ do volume total, na parte central e superior do vaso ("cova"), com sete doses de P nos $4,77 \mathrm{dm}^{3} \mathrm{de}$ solo circundante ("externo") (Figura 1). Foram utilizadas as 13 combinações da matriz experimental "Box - Berard aumentada (3)" (Leite, 1984), mais dois tratamentos adicionais: um, em cuja combinação a dose de P da "cova" foi menor que a dose do solo "externo" (T14), e outro, em que a combinação correspondente ao ponto central da matriz utilizada $(0,00: 0,00)$ foi repetida, porém sem incubação e com o P do solo "externo" localizado (T15) (Figura 1 e Quadro 2).

As doses de P (Quadro 2), nos dois solos, foram escolhidas dentro de diferentes espaços experimentais, por apresentarem diferente capacidade tampão (Alvarez V. \& Fonseca, 1990). As doses usadas no solo LVAm-LS correspondem às doses utilizadas no solo LVAd-VI divididas por 1,75. As quantidades totais de $\mathrm{P}$ aplicadas por vaso variaram de acordo com a combinação de doses.

Os 15 tratamentos foram distribuídos em blocos casualizados com cinco repetições.

As doses de N, de $\mathrm{S}$, de $\mathrm{K}$ e a calagem foram iguais no solo da "cova" e no solo "externo". Foram utilizados, como fontes de $\mathrm{P}$, o superfosfato simples, o superfosfato triplo, $\mathrm{KH}_{2} \mathrm{PO}_{4}$ e $\mathrm{NH}_{4} \mathrm{H}_{2} \mathrm{PO}_{4}$, em quantidades limitadas pelas doses dos nutrientes acompanhantes. Os micronutrientes foram fornecidos durante o ensaio, em forma de solução.

Os volumes de solo, correspondentes à "cova" e ao solo "externo", foram incubados individualmente com calcário por $20 \mathrm{~d}$. Após a secagem, os solos receberam as doses de $\mathrm{P}$ e demais nutrientes e foram incubados, a $80 \%$ da capacidade de campo, por mais $40 \mathrm{~d}$. Ao final da incubação e após a retirada de amostras para a determinação de P, os solos da "cova" e "externo" foram acondicionados nos vasos (Figura 1).

Cada vaso recebeu, então, quatro sementes de milho híbrido (Pioneer 3210) em sua parte média ("cova"), das quais, após desbaste, cultivaram-se duas plantas.

Aos $32 \mathrm{~d}$ da emergência, fez-se o corte da parte aérea, que foi seca em estufa de ventilação forçada, a $70^{\circ} \mathrm{C}$, para determinação da produção de matéria seca (MS). Em duas das cinco repetições, retiraram-se amostras representativas da parte superior dos vasos, incluindo "cova" e solo "externo" $\left(\mathrm{C}+\mathrm{E}_{1}\right.$, Figura 1), para determinação de $\mathrm{P}$ (Mehlich-1).

Considerou-se, como índice de eficiência do $\mathrm{P}$ adicionado ao vaso, a quantidade de matéria seca da parte aérea produzida por unidade de $\mathrm{P}$ adicionado (mg de MS por mg de $\mathrm{P}$ aplicado).

As equações de regressão foram selecionadas após a análise de variância, testando-se os coeficientes dos componentes de cada modelo até $10 \%$ de probabilidade

Quadro 1. Características químicas e físicas das amostras dos solos utilizadas no experimento(1)

\begin{tabular}{|c|c|c|}
\hline \multirow{2}{*}{ Característica } & \multicolumn{2}{|c|}{ Solo } \\
\hline & LVAd-VI & LVAm-LS \\
\hline $\mathrm{pH}\left(\mathrm{H}_{2} \mathrm{O}, 1: 2,5\right)$ & 4,40 & 4,70 \\
\hline $\mathrm{Al}^{3+}\left(\mathrm{cmol}_{\mathrm{c}} \mathrm{dm}^{-3}\right)^{(2)}$ & 1,00 & 0,60 \\
\hline $\mathrm{Ca}^{2+}\left(\mathrm{cmol}_{\mathrm{c}} \mathrm{dm}^{-3}\right)^{(2)}$ & 0,10 & 0,30 \\
\hline $\mathrm{Mg}^{2+}\left(\mathrm{cmol}_{\mathrm{c}} \mathrm{dm}^{-3}\right)^{(2)}$ & 0,04 & 0,03 \\
\hline $\mathrm{H}+\mathrm{Al}\left(\mathrm{cmol}_{\mathrm{c}} \mathrm{dm}^{-3}\right)^{(3)}$ & 5,70 & 3,60 \\
\hline $\mathrm{P}\left(\mathrm{mg} \mathrm{dm}^{-3}\right)^{(4)}$ & 1,00 & 0,70 \\
\hline $\mathrm{K}\left(\mathrm{mg} \mathrm{dm}^{-3}\right)^{(4)}$ & 9,00 & 36,00 \\
\hline P rem $\left(\mathrm{mg} \mathrm{L}^{-1}\right)^{(5)}$ & 4,00 & 16,00 \\
\hline C. Orgânico (dag kg-1) & 2,00 & 1,10 \\
\hline Capacidade de campo $\left(\mathrm{kg} \mathrm{kg}^{-1}\right)$ & 0,32 & 0,22 \\
\hline Areia Grossa (\%) & 13,00 & 44,00 \\
\hline Areia Fina (\%) & 16,00 & 17,00 \\
\hline Silte (\%) & 5,00 & 6,00 \\
\hline Argila (\%) & 66,00 & 33,00 \\
\hline Classificação textural & Iuito Argilos & Argiloso A \\
\hline
\end{tabular}

$\overline{(1)}$ LVAd-VI = Latossolo Vermelho-Amarelo distrófico, do Tiro de Guerra de Viçosa-MG; LVAm-LS = Latossolo VermelhoAmarelo, de Lassance-MG. ${ }^{(2)}$ Extrator $\mathrm{KCl} 1 \mathrm{~mol} \mathrm{~L}^{-1} .{ }^{(3)}$ Extrator Mehlich-1. ${ }^{(4)}$ Extrator $\mathrm{Ca}(\mathrm{OAc})_{2} 0,5 \mathrm{~mol} \mathrm{~L}{ }^{-1}, \mathrm{pH} 7 .{ }^{(5)} \mathrm{P}$ rem $=$ Fósforo remanescente (Alvarez V. et al., 2000). 
(a)

(a) $6,80 \mathrm{~cm}$
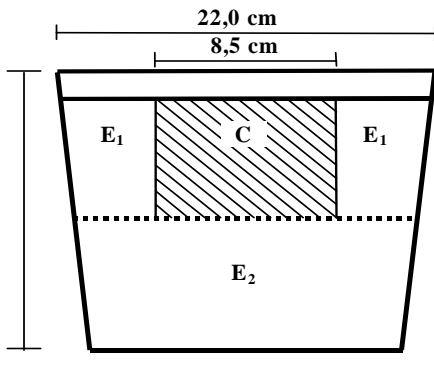

$17,8 \mathrm{~cm}$

WW Solo “cova” (C)

Solo “externo" (E)

(b)

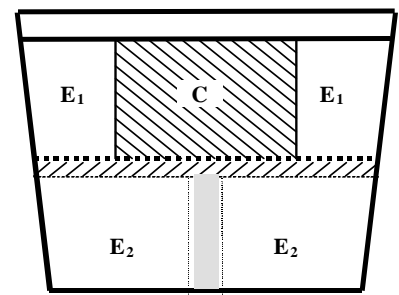

WW Solo “cova” (C)

Solo “externo” (E)

DDPDT Localização $\mathrm{PE}_{1}$ (Superfosfato simples)

Localização $P E_{1}$ (Superfosfato triplo)
Volume $\left(\mathrm{dm}^{3}\right)$ :

$\mathrm{C}=\quad 0,53$

$E_{1}=\quad 2,77$

$E_{2}=\frac{2,00}{5,30}$
Volume $\left(\mathrm{dm}^{3}\right)$ :

$\mathrm{C}=\quad 0,53$

$E_{1}=2,77$

$E_{2}=\frac{2,00}{5,30}$

Figura 1. Localização do $\mathrm{P}$ nos 14 primeiros tratamentos (a) e no $15^{\mathrm{o}}$ tratamento (b).

Quadro 2. Doses de P para a "cova" (C) e para o solo "externo" (E) e quantidades de P aplicadas por vaso, nas amostras dos solos LVAd-VI e LVAm-LS

\begin{tabular}{|c|c|c|c|c|c|}
\hline \multirow{2}{*}{\multicolumn{2}{|c|}{ Tratamento $\mathrm{N}^{\circ}$}} & \multicolumn{2}{|c|}{ Solo LVAd-VI } & \multicolumn{2}{|c|}{ Solo LVAm-LS ${ }^{(3)}$} \\
\hline & & \multirow{2}{*}{$\begin{array}{c}\text { Dose de P } \\
C+E\end{array}$} & \multirow{2}{*}{$\begin{array}{c}\text { P aplicado } \\
\text { por vaso }\end{array}$} & \multirow{2}{*}{$\begin{array}{c}\text { Dose de P } \\
C+E\end{array}$} & \multirow{2}{*}{$\begin{array}{c}P \text { aplicado } \\
\text { por vaso }\end{array}$} \\
\hline $\mathbf{N}^{0}$ & $\mathbf{C}+\mathbf{E}$ & & & & \\
\hline & & $\mathrm{mg} \mathrm{dm} \mathrm{m}^{-3}$ & $\mathrm{mg}$ vaso $^{-1}$ & $\mathrm{mg} \mathrm{dm} \mathrm{m}^{-3}$ & $\mathrm{mg} \mathrm{vaso}^{-1}$ \\
\hline $\mathrm{T} 1$ & $-0,50:-0,50$ & $175,0+35,0$ & 259,7 & $100,0+20,0$ & 148,4 \\
\hline $\mathrm{T} 2$ & $-0,50: 0,50$ & $175,0+105,0$ & 593,6 & $100,0+60,0$ & 339,2 \\
\hline $\mathrm{T} 3$ & $0,50:-0,50$ & $525,0+35,0$ & 445,2 & $300,0+20,0$ & 254,4 \\
\hline $\mathrm{T} 4$ & $0,50: 0,50$ & $525,0+105,0$ & 779,1 & $300,0+60,0$ & 445,4 \\
\hline T5 & $0,00: 0,00$ & $350,0+70,0$ & 519,4 & $200,0+40,0$ & 296,8 \\
\hline T6 & $-0,75: 0,00$ & $87,5+70,0$ & 380,3 & $50,0+40,0$ & 217,3 \\
\hline $\mathrm{T} 7$ & $0,75: 0,00$ & $612,5+70,0$ & 658,5 & $350,0+40,0$ & 376,3 \\
\hline $\mathrm{T} 8$ & $0,00:-0,75$ & $350,0+17,5$ & 269,0 & $200,0+10,0$ & 153,7 \\
\hline T9 & $0,00: 0,75$ & $350,0+122,5$ & 769,8 & $200,0+70,0$ & 439,9 \\
\hline $\mathrm{T} 10$ & $-0,90:-0,50$ & $35,0+35,0$ & 185,5 & $20,0+20,0$ & 106,0 \\
\hline $\mathrm{T} 11$ & $-0,50:-0,90$ & $175,0+7,0$ & 126,1 & $100,0+40,0$ & 72,1 \\
\hline $\mathrm{T} 12$ & $0,90: 0,50$ & $665,0+105,0$ & 853,3 & $380,0+60,0$ & 487,6 \\
\hline $\mathrm{T} 13$ & $0,50: 0,90$ & $525,0+133,0$ & 912,7 & $300,0+76,0$ & 521,5 \\
\hline $\mathrm{T} 14$ & $-0,90: 0,90^{(1)}$ & $35,0+133,0$ & 653,0 & $20,0+76,0$ & 374,2 \\
\hline $\mathrm{T} 15$ & $0,00: 0,00 \mathrm{~L}^{(2)}$ & $350,0+70,0 \mathrm{~L}$ & 519,4 & $200,0+40,0 \mathrm{~L}$ & 296,8 \\
\hline
\end{tabular}

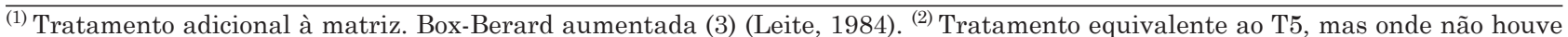
incubação antes do plantio e a dose de P do solo "externo" foi localizada (L). ${ }^{(3)}$ As doses usadas no solo LVAm-LS correspondem às doses utilizadas no solo LVAd-VI divididas por 1,75. 
Foram escolhidos os modelos significativos com maior coeficiente de determinação $\left(\mathrm{R}^{2}\right)$.

\section{RESULTADOS E DISCUSSÃO}

\section{Fósforo disponível no solo e produção de matéria seca da parte aérea}

Os teores de $\mathrm{P}$ nos dois solos foram semelhantes, apesar de as doses aplicadas ao solo LVAd-VI terem sido maiores (Quadro 3). Isso reflete o maior desgaste do extrator Mehlich-1 no solo de maior poder tampão (LVAd-VI - muito argiloso), em relação ao solo LVAmLS (textura média), como discutido por Holford (1997) e por Novais \& Smyth (1999).

As equações de regressão para os teores de $\mathrm{P}$ recuperados da "cova" e do solo "externo", considerando as doses aplicadas às respectivas regiões, apresentaram excelente grau de ajuste (Quadro 4).

A forma de localização do P no solo influenciou, acentuadamente, a produção de MS da parte aérea das plantas de milho (Quadro 5). Além de explicar claramente as variações de crescimento, a localização

Quadro 3. Teores de P disponível pelo extrator Mehlich-1 após incubação dos solos com as doses de $\mathbf{P}$ aplicadas na "cova" (Pc) e na parte "externa" (Pe)

\begin{tabular}{|c|c|c|c|c|c|}
\hline \multicolumn{2}{|c|}{ Tratamento $\mathrm{N}^{\circ}$} & \multicolumn{2}{|c|}{ Solo LVAd-VI } & \multicolumn{2}{|c|}{ Solo LVAm-LS } \\
\hline $\mathbf{N}^{\mathbf{o}}$ & $\mathbf{C}+\mathbf{E}$ & Cova & Externo & Cova & Externo \\
\hline $\mathrm{T} 1$ & $-0,50:-0,50$ & 22,60 & 3,91 & 23,64 & 4,25 \\
\hline $\mathrm{T} 2$ & $-0,50: 0,50$ & 22,24 & 11,10 & 22,81 & 10,94 \\
\hline T3 & $0,50:-0,50$ & 92,18 & 3,43 & 91,91 & 3,90 \\
\hline $\mathrm{T} 4$ & $0,50: 0,50$ & 93,28 & 11,88 & 95,38 & 11,57 \\
\hline T5 & $0,00: 0,00$ & 52,93 & 7,27 & 60,85 & 8,23 \\
\hline $\mathrm{T} 6$ & $0,75: 0,00$ & 9,27 & 7,61 & 10,73 & 7,49 \\
\hline $\mathrm{T} 7$ & $-0,75: 0,00$ & 106,03 & 7,03 & 107,99 & 8,36 \\
\hline $\mathrm{T} 8$ & $0,00:-0,75$ & 52,27 & 1,91 & 60,34 & 2,43 \\
\hline T9 & $0,00: 0,75$ & 55,25 & 15,28 & 58,69 & 14,15 \\
\hline $\mathrm{T} 10$ & $-0,90:-0,50$ & 3,98 & 3,50 & 4,20 & 3,75 \\
\hline $\mathrm{T} 11$ & $-0,50:-0,90$ & 22,95 & 1,27 & 22,97 & 1,77 \\
\hline $\mathrm{T} 12$ & $0,90: 0,50$ & 130,60 & 10,84 & 115,08 & 11,67 \\
\hline $\mathrm{T} 13$ & $0,50: 0,90$ & 91,61 & 16,65 & 104,93 & 15,57 \\
\hline $\mathrm{T} 14$ & $-0,90: 0,90$ & 3,50 & 16,87 & 4,63 & 15,54 \\
\hline $\mathrm{T} 15$ & $0,00: 0,00 L^{(1)}$ & - & - & - & - \\
\hline
\end{tabular}

Quadro 4. Equações de regressão para os teores de $\mathrm{P}$ recuperado $\left(\mathrm{Pr}, \mathrm{em} \mathrm{mg} \mathrm{dm}^{-3} \mathrm{de} \mathrm{P}\right)$ pelo Extrator Mehlich-1 do solo da "cova" e do solo "externo", após incubação e antes do plantio do milho, como variável dependente das doses de $\mathrm{P}\left(\mathrm{mg} \mathrm{dm}^{-3} \mathrm{de} \mathrm{P}\right)$, aplicadas na "cova" (Pc) ou no solo "externo" (Pe), nos solos LVAd-VI e LVAm-LS

\begin{tabular}{|c|c|c|}
\hline Localização & Equação & $\mathbf{R}^{2}$ \\
\hline \multicolumn{3}{|c|}{ Solo LVAd-VI } \\
\hline Cova & $\hat{\mathrm{P}} \mathrm{r}=-0,707+0,113095^{* *} \mathrm{Pc}+0,0001183^{* *} \mathrm{Pc}^{2}$ & 0,997 \\
\hline Externo & $\hat{\mathrm{P}} \mathrm{r}=1,101+0,049916^{* *} \mathrm{Pe}+0,0005000^{* *} \mathrm{Pe}^{2}$ & 0,993 \\
\hline \multicolumn{3}{|c|}{ Solo LVAm-LS } \\
\hline Cova & $\hat{\mathrm{P}} \mathrm{r}=1,791-1,48724^{* *} \mathrm{Pc}^{1 / 2}+0,391108^{* *} \mathrm{Pc}$ & 0,990 \\
\hline Externo & $\hat{\mathrm{P}} \mathrm{r}=1,066+0,139339 * * \mathrm{Pe}+0,0006492 * \mathrm{Pe}^{2}$ & 0,994 \\
\hline
\end{tabular}


permitiu obter elevados $R^{2}$ para as equações de regressão da produção de MS, em funçâo das doses de $\mathrm{P}$ aplicadas à "cova" $(\mathrm{Pc})$ e ao "solo externo" $(\mathrm{Pe})$ (Quadro 6). Entretanto, houve pequena relação (baixo $\mathrm{R}^{2}$ ) da produção com a quantidade total de $\mathrm{P}$ aplicada ao vaso (Pav) (Quadro 6). Assim, com a aplicação de quantidades semelhantes de $\mathrm{P}$ por vaso, foram obtidas produções de MS muito diferentes, pela modificação dos teores de $\mathrm{P}$ dentro dos vasos, de acordo com as doses usadas localizadamente na "cova" e no solo "externo".

A resposta para as doses na "cova", no intervalo de doses baixas, foi muito pequena, mas, a partir de certa faixa de doses, esta passou a ser muito alta, limitando a resposta ao P aplicado no solo "externo", que não chegou a atingir o máximo no intervalo utilizado (Quadro 6).

Nos dois solos, a resposta a Pe ocorreu apenas na presença de doses baixas de Pc, em que o crescimento das plantas de milho foi pequeno (Quadro 6), contrariando observações da literatura. Segundo Welch et al. (1966), para a obtenção de altas produções de milho, não basta uma simples aplicação de $\mathrm{P}$ no sulco de plantio, sendo necessária aplicação suplementar a lanço. Para que haja resposta do milho à adubação no sulco, deve haver uma disponibilidade pelo menos razoável no restante da camada arável (Novais et al., 1985; Klepker \& Anghinoni, 1995; Alves et al., 1999).

Quadro 5. Produção de matéria seca da parte aérea de plantas de milho (MS), de acordo com as doses de $\mathbf{P}$ aplicadas na "cova" (Pc) e no solo "externo" (Pe), quantidades totais aplicadas no vaso (Pav) e eficiência de utilização do P adicionado nos solos LVAd-VI e LVAm-LS

\begin{tabular}{|c|c|c|c|c|c|c|c|}
\hline \multicolumn{2}{|c|}{ Tratamento $\mathrm{N}^{\mathrm{o}}$} & \multicolumn{3}{|c|}{ Solo LVAm-VI } & \multicolumn{3}{|c|}{ Solo LVAm-LS } \\
\hline $\mathrm{N}^{\mathrm{o}}$ & $\mathbf{C}+\mathbf{E}$ & $P$ aplicado & Matéria seca & Efi.do PAd. & P aplicado & Matéria seca & Efi.do PAd. \\
\hline & & $\mathrm{mg}$ vaso $^{-1}$ & g vaso-1 & $\mathrm{mg} \mathrm{mg}^{-1}$ & $\mathrm{mg}$ vaso $^{-1}$ & g vaso $^{-1}$ & $\mathrm{mg} \mathrm{mg}^{-1}$ \\
\hline $\mathrm{T} 1$ & $-0,50:-0,50$ & 259,7 & 2,14 & 8,3 & 148,4 & 2,05 & 13,8 \\
\hline $\mathrm{T} 2$ & $-0,50: 0,50$ & 593,6 & 2,50 & 4,2 & 339,2 & 3,40 & 10,0 \\
\hline T3 & $0,50:-0,50$ & 445,2 & 8,82 & 19,8 & 254,4 & 6,00 & 23,6 \\
\hline $\mathrm{T} 4$ & $0,50: 0,50$ & 779,1 & 7,76 & 10,0 & 445,4 & 6,46 & 14,5 \\
\hline T5 & $0,00: 0,00$ & 519,4 & 4,00 & 7,7 & 296,8 & 4,55 & 15,3 \\
\hline T6 & $-0,75: 0,00$ & 380,3 & 1,70 & 4,5 & 217,3 & 1,96 & 9,0 \\
\hline $\mathrm{T} 7$ & $0,75: 0,00$ & 658,5 & 9,35 & 14,2 & 376,3 & 7,78 & 20,7 \\
\hline $\mathrm{T} 8$ & $0,00:-0,75$ & 269,0 & 3,30 & 12,3 & 153,7 & 4,08 & 26,5 \\
\hline T9 & $0,00: 0,75$ & 769,8 & 4,46 & 5,8 & 439,9 & 4,35 & 9,9 \\
\hline T10 & $-0,90:-0,50$ & 185,5 & 1,36 & 7,3 & 106,0 & 1,45 & 13,6 \\
\hline $\mathrm{T} 11$ & $-0,50:-0,90$ & 126,1 & 1,74 & 13,8 & 72,1 & 1,92 & 26,7 \\
\hline T12 & $0,90: 0,50$ & 853,3 & 11,66 & 13,7 & 487,6 & 8,20 & 16,8 \\
\hline $\mathrm{T} 13$ & $0,50: 0,90$ & 912,7 & 7,67 & 8,4 & 521,5 & 7,75 & 14,9 \\
\hline $\mathrm{T} 14$ & $-0,90: 0,90$ & 653,0 & 2,37 & 3,6 & 374,2 & 3,26 & 8,7 \\
\hline \multirow[t]{2}{*}{$\mathrm{T} 15$} & $0,00: 0,00 \mathrm{~L}^{(1)}$ & 519,4 & $8,29 * *$ & 16,0 & 296,8 & $11,09 * *$ & 37,4 \\
\hline & Média & 528,4 & 5,16 & 10,0 & 308,6 & 4,95 & 17,4 \\
\hline
\end{tabular}

(1) Tratamento adicional em que não houve incubação e a dose Pe foi localizada.

** = Diferente significativamente do tratamento equivalente (T5 no solo LVAd-VI e no solo LVAm-LS) a $1 \%$.

Quadro 6. Equações de regressão da produção de matéria seca da parte aérea (MS) de plantas de milho ( g vaso $\left.^{-1}\right)$, como variável dependente das doses de $\mathrm{P}\left(\mathrm{mg} \mathrm{dm}^{-3}\right)$, aplicadas na "cova" (Pc) e no "solo externo" (Pe), das quantidades totais de $\mathrm{P}$, aplicadas no vaso (Pav, em $\mathrm{mg}^{\text {vaso }}{ }^{-1}$ ), nos solos LVAd-VI e LVAm-LS

\section{Equação}

$\mathbf{R}^{2}$

Solo LVAd-VI

$\hat{\mathrm{M}} \mathrm{S}=0,864-0,00013 \mathrm{Pc}+0,01260 * * \mathrm{Pe}+0,000026^{* *} \mathrm{Pc}^{2}-0,000027^{*} \mathrm{PcPe} \quad 0,985$

$\hat{\mathrm{M} S}=1,230+0,003496^{\circ} \mathrm{Pav}+0,00000054^{* *} \mathrm{Pav}^{2} \quad 0,468$

Solo LVAm-LS

$\hat{\mathrm{M} S}=1,622-0,26625^{*} \mathrm{Pc}^{1 / 2}-0,03956 \mathrm{Pe}^{1 / 2}+0,03275^{* *} \mathrm{Pc}+0,04215^{\circ} \mathrm{Pe}-0,01720^{\circ}(\mathrm{Pc} \mathrm{Pe})^{1 / 2} \quad 0,982$

$\hat{\mathrm{M}} \mathrm{S}=1,396+0,006417 * \mathrm{Pav}+0,0000106 * \mathrm{Pav}^{2}$

0,627

${ }^{\circ}, *, * *$ : Significativos a 10,5 e $1 \%$, respectivamente. 
Para se ter melhor idéia do efeito da localização do $\mathrm{P}$, podem-se comparar as respostas no solo LVAd-VI dos tratamentos T14 $\left(35+133 \mathrm{mg} \mathrm{dm}^{-3}\right)$, T5 $(350+70)$, T3 $(525+35)$ e T15 $\left(350+70 \mathrm{~L} \mathrm{mg} \mathrm{dm}^{-3}\right)$. O tratamento adicional T14 $\left(35+133 \mathrm{mg} \mathrm{dm}^{-3} \mathrm{na}\right.$ "cova" e no solo "externo") apresentou produção de MS da parte aérea das plantas de milho de $2,37 \mathrm{~g}^{\text {vaso }^{-1} \mathrm{e}}$ eficiência de utilização do $\mathrm{P}$ adicionado de $3,6 \mathrm{mg}$ de MS por $\mathrm{mg}$ de $\mathrm{P}$, com adição de $653 \mathrm{mg}^{\mathrm{vaso}}{ }^{-1} \mathrm{de} \mathrm{P}$. A produção de MS do tratamento T5 (350-70) foi de $4,00 \mathrm{~g}^{\text {vaso }^{-1}}$, ou seja, $69 \%$ a mais do que o T14, com uso de menor quantidade de $\mathrm{P}\left(519,4 \mathrm{mg} \mathrm{vaso}^{-1}\right)$, que também aumentou a eficiência do $\mathrm{P}$ adicionado, eficiência que foi de 7,7 $\mathrm{mg}$ de MS por $\mathrm{mg}$ de $\mathrm{P}$ (Quadro 5). O tratamento T5 $(350+70)$ concentra a localização do P na "cova" 10 vezes em relação ao T14 $(35+133)$ e diminui a dose no solo "externo". Para entender melhor esta localização, observar os efeitos dos tratamentos T3 $(525+35)$ e T15 $(350+70 \mathrm{~L})$. O tratamento T3, em que se adicionaram unicamente 445,2 $\mathrm{mg}$ vaso $^{-1}$ de $\mathrm{P}$, apresentou produção de $8,82 \mathrm{~g}$ vaso $^{-1}$ de MS de plantas de milho, um aumento de $272 \%$ em relação à resposta obtida no tratamento T14 (35 + 133). Também a eficiência do $\mathrm{P}$ adicionado foi bem maior: 19,8 $\mathrm{mg}$ de MS por $\mathrm{mg}$ de P. O tratamento adicional T15 (350-70L), com doses semelhantes às do $\mathrm{T} 5$, mas sem incubação e em que a dose do solo "externo" também foi localizada (Figura 1B), apresentou produção de $8,29 \mathrm{~g}$ vaso $^{-1} \mathrm{de}$ MS e eficiência de utilização do $P$ adicionado de $16 \mathrm{mg}$ de MS por mg de P. A produção aumentou $107 \%$ em relação ao T5, em razão da falta de incubação e da localização da dose de P no solo "externo" (Quadro 5).

Resultados semelhantes podem ser observados no solo LVAm-LS em relação à produção de MS, mas com maiores aumentos na eficiência do $\mathrm{P}$ adicionado (Quadro 5), por terem sido usadas menores doses de P e por apresentar este solo menor capacidade tampão para $\mathrm{P}$.

De modo geral, maiores eficiências no aproveitamento do $\mathrm{P}$ adicionado foram obtidas com a aplicação de maiores doses de P ao solo da "cova" (Quadro 5), retratando a influência da forma de localização do $\mathrm{P}$ sobre seu aproveitamento. Segundo Randall e Hoeft
(1988), em solos pobres em P, a localização na zona radicular efetiva geralmente resulta em maior eficiência no uso do P. Para Sleight et al. (1984), o uso mais eficiente do $\mathrm{P}$ pelas plantas jovens se dá quando o nutriente é colocado no solo, próximo à semente, onde haverá alto crescimento de raízes.

\section{Doses recomendáveis de fósforo}

Como a resposta para Pe foi insignificante, adotouse a menor dose do espaço experimental estudado como sendo a recomendável para o solo "externo". Como não foram atingidas produções máximas no espaço fatorial, para a "cova", adotou-se como a dose Pc recomendável à necessária para a obtenção de $90 \%$ da produção obtida com a maior dose, junto com a menor dose de Pe (Quadro 7).

Caso a quantidade total de $\mathrm{P}$ das doses recomendáveis para as duas regiões fosse aplicada uniformemente ao volume de solo do vaso $\left(5,3 \mathrm{dm}^{3}\right)$ e não de forma localizada ("cova", solo "externo"), a produção estimada com as equações do quadro 6 seria de $1,77 \mathrm{~g} \mathrm{vaso}^{-1}$, no solo LVAd-VI, e de 1,94 g vaso- ${ }^{-1}$, no solo LVAmLS. Portanto, bem abaixo das produções estimadas (Quadro 7).

Este critério de localização de $\mathrm{P}$ aumenta a eficiência de aproveitamento do $\mathrm{P}$ adicionado por várias razões. A colocação do $P$ próximo à semente aumenta a chance do contato raiz:P e diminui o tempo para que esse contato ocorra (Sleight et al., 1984). Todas as raízes iniciais do milho atravessaram a zona com alto teor de $\mathrm{P}$ e o contato do maior volume de raízes possível com o P é condição para maior crescimento do milho (Jungk \& Barber, 1974; Striker et al., 1974 ; Novais et al., 1985). Assim, garante-se alto teor de P na fase inicial de crescimento da planta, na qual a demanda de P pela mesma é alta, como verificado para trigo (Romer e Schiling, 1986), para eucalipto (Neves, 1983) e para o próprio milho (Anghinoni, 1992).

\section{Níveis críticos de fósforo na "cova" e no "solo externo"}

Foram calculados os níveis críticos de P na "cova" e no solo "externo" (Quadro 8), a partir das respectivas

Quadro 7. Doses recomendáveis de P para o solo da "cova" e solo "externo" para a obtenção de 90 \% da maior produção de matéria seca (MS) da parte aérea de milho, quantidade equivalente de $P$ a ser aplicada no vaso e eficiência de utilização do $P$ adicionado nos solos LVAd-VI e LVAm-LS

\begin{tabular}{|c|c|c|c|c|}
\hline \multicolumn{2}{|c|}{ Dose recomendável de $\mathbf{P}$} & \multirow{2}{*}{$\begin{array}{c}\text { P a ser aplicado } \\
\text { no vaso }\end{array}$} & \multirow{2}{*}{$\begin{array}{l}90 \% \text { da maior } \\
\text { produção de MS }\end{array}$} & \multirow{2}{*}{$\begin{array}{c}\text { Eficiência de utilização } \\
\text { do P Adicionado }\end{array}$} \\
\hline Cova & Externo & & & \\
\hline \multirow{2}{*}{\multicolumn{2}{|c|}{$\mathrm{mg} \mathrm{mg}^{-1}$}} & \multicolumn{2}{|c|}{$\mathrm{mg} \mathrm{vaso}^{-1}$} & $\mathrm{mg} \mathrm{mg}^{-1}$ \\
\hline & & \multicolumn{2}{|c|}{ Solo LVAm-LS } & \\
\hline 627,88 & 7,00 & 366,2 & 10,85 & 29,6 \\
\hline \multicolumn{5}{|c|}{ Solo LVAd-VI } \\
\hline 346,60 & 4,00 & 202,8 & 7,47 & 36,8 \\
\hline
\end{tabular}


doses recomendáveis, substituindo-se os valores dessas doses nas equações para os teores de $\mathrm{P}$ recuperados do solo pelo extrator Mehlich-1, em função das doses aplicadas (Quadro 4).

Os elevados valores de nível crítico de $\mathrm{P}$ obtidos para a "cova" (Quadro 8) estão de acordo com resultados obtidos em casa de vegetação, com a aplicação uniforme do $\mathrm{P}$ em todo o volume do solo. Os resultados obtidos nesses trabalhos envolviam aplicações de grandes quantidades de $\mathrm{P}$ para corrigir o volume total de solo e atender às exigências da planta. Com a aplicação localizada ("cova”), o valor de nível crítico, que representa essa região, tenderá a ser ainda maior. No entanto, como a fração de volume de solo a ser fertilizada é pequena, a quantidade a ser aplicada não será grande.

Como na obtenção de altas produções praticamente não houve resposta ao P do solo "externo", adotou-se, para critério de recomendação, a fixação de um valor

Quadro 8. Níveis críticos de P do solo da "cova" e do solo "externo", pelo extrator Mehlich-1, para a obtenção de 90 \% da produção máxima da parte aérea de plantas de milho nos solos LVAd-VI e LVAm-LS

\begin{tabular}{ccc}
\hline \multirow{2}{*}{ Solo } & \multicolumn{2}{c}{ Solo } \\
\cline { 2 - 3 } & Cova & Externo \\
\hline & \multicolumn{2}{c}{$\mathrm{mg} \mathrm{dm}^{-3}-$} \\
LVAd-VI & 116,9 & 1,5 \\
LVAm-LS & 109,7 & 1,6 \\
\hline
\end{tabular}

de nível crítico somente para a "cova". Dessa forma, por simulação, para quaisquer teores do P original do solo, a recomendação de adubação, a ser aplicada na "cova", será aquela que eleve o teor de $\mathrm{P}$, nessa região, para o valor do nível crítico (Quadro 9).

Estimou-se, então, a dose de $\mathrm{P}$ a ser recomendada (DR) para a "cova", para cada teor de P original do solo, empregando-se a fórmula:

$$
\mathrm{DR}=(\mathrm{NiCri}-\mathrm{Pd}) / \Delta(\operatorname{Prp} / \mathrm{Pad})
$$

em que: NiCri é o nível crítico estimado para a "cova" (Quadro 8); Pd é o teor de P disponível em condições de solos de baixa, média e alta fertilidade, dentro do intervalo experimental de doses para o solo "externo" e $\Delta(\operatorname{Prp} / \mathrm{Pad})$ é a declividade obtida da equação de $\mathrm{P}$ disponível, em função da dose de $\mathrm{P}$ adicionada à "cova".

As doses a serem recomendadas e as quantidades de $\mathrm{P}$ a serem adicionadas diminuem à medida que cresce o teor de $\mathrm{P}$ do solo original, pois menor será a quantidade de $\mathrm{P}$ necessária para que se alcance o nível crítico (Quadro 9).

Os teores de $\mathrm{P}$ recuperado da mistura do solo da parte superior do vaso (Prs), parte do solo "externo" mais a "cova" $\left(\mathrm{C}+\mathrm{E}_{1}\right.$, Figura 1) após a colheita do milho, foram relacionados com as doses de $\mathrm{P}$ aplicadas na "cova" e no solo "externo" (Pc e Pe) e com as quantidades de $\mathrm{P}$ aplicadas em todo o solo do vaso (Pav) ou no solo da parte superior do mesmo (Pas) (Quadro 10).

Os elevados valores de $\mathrm{R}^{2}$ obtidos para as equações (Quadro 10) mostram que essa forma de amostragem foi representativa para a determinação do teor médio de $\mathrm{P}$ do volume de solo amostrado. Em áreas que receberam adubação localizada de $\mathrm{P}$, recomenda-se a amostragem do solo após o preparo mecânico do terreno,

Quadro 9. Doses a serem recomendadas e quantidades de $P$ a serem aplicadas na "cova" $\left(0,53 \mathrm{dm}^{3}\right) \mathrm{em} \mathrm{solos}^{\circ}$ com três diferentes teores de $\mathrm{P}$ disponíveis para atingir o nível crítico para milho nos solos LVAd-VI e LVAm-LS

Teor de P no solo Nível crítico de P na cova $\mathbf{P}^{(1)} \quad \begin{gathered}\text { Dose de P a ser } \\ \text { recomendada na cova }\end{gathered} \quad$ P a ser aplicado na cova

\begin{tabular}{|c|c|c|c|}
\hline \multicolumn{4}{|c|}{ Solo LVAd-VI } \\
\hline 1,5 & 115,0 & 615,0 & 326,0 \\
\hline 7,0 & 115,0 & 593,0 & 314,3 \\
\hline 16,5 & 115,0 & 554,0 & 293,6 \\
\hline \multicolumn{4}{|c|}{ Solo LVAm-LS } \\
\hline 1,5 & 110,0 & 343,0 & 181,8 \\
\hline 7,0 & 110,0 & 325,0 & 172,3 \\
\hline 16,5 & 110,0 & 303,0 & 160,6 \\
\hline
\end{tabular}

(1) Níveis críticos definidos para atingir $90 \%$ da produção de matéria seca da parte aérea de plantas de milho, obtida com a maior dose de P aplicada na "cova". 
pois ocorre mistura do solo e, portanto, dos teores de $\mathrm{P}$, uma vez que estes podem variar de acordo com as proporções de terra provenientes do sulco e das entrelinhas (Vasconcelos et al., 1982).

Entretanto, no plantio convencional (aração/ gradagem), a amostragem de solo é geralmente realizada antes da aração, visando a uma possível incorporação de corretivos da acidez e, no plantio direto, por princípio, não se promove a mistura do solo. Nestas condições, para suplantar a elevada variabilidade causada pela localização do adubo fosfatado, pode-se utilizar a amostragem com pá de corte, ou seja, fatia de solo que encerra mistura dos teores contidos no sulco e nas entrelinhas (Schlindwein \& Anghinoni, 1998). Outra alternativa é fazer a coleta proporcional e direcionada de amostras simples no sulco e nas entrelinhas (Alvarez V., 1982 ; Nicolodi et al., 2002).

A forma de amostragem deve considerar, também, a exploração do volume de solo pela planta e sua resposta em termos de crescimento. Os $\mathrm{R}^{2}$ para a produção de MS em função dos teores de $\mathrm{P}$ recuperado após o plantio (Prs) são baixos (Quadro 11), quando comparados com aqueles obtidos nas equações de MS em função de Pc e Pe (Solo LVAd-VI: $\mathrm{R}^{2}=0,985$ e solo LVAm-LS: $\mathrm{R}^{2}=0,982$, Quadro 6), mostrando que os teores de $\mathrm{P}$ obtidos podem ser representativos do volume cultivado, mas não representam bem a disponibilidade efetiva para o crescimento inicial das plantas nesse volume de solo. A importância do ajuste da forma de amostragem do solo, em condições de adubação localizada em cana-de-açúcar, no sentido de representar melhor a disponibilidade de $\mathrm{P}$ do solo explorado pelas raízes da cultura, foi levantada por Genta \& Carnelli (1983).

Substituindo as doses de Pc e Pe recomendáveis ou a quantidade equivalente de $\mathrm{P}$ aplicada no vaso (Pav) ou ao volume amostrado (Pas), nas respectivas equações para $\mathrm{P}$ recuperado do solo, após a colheita do milho, foram obtidos os níveis críticos, ou seja, os teores de $\mathrm{P}$ no solo relacionados com 90 \% da maior produção de MS (Quadro 12).

Quadro 10. Equações de regressão dos teores de $P$ recuperado pelo extrator Mehlich-1 $\left(\mathrm{mg} \mathrm{dm}^{-3} \mathrm{de} P\right) \mathrm{da}$ mistura do solo da parte superior do vaso (Prs), "cova" mais solo "externo" $\left(\mathrm{C}+\mathrm{E}_{1}\right)$, amostrada após a colheita do milho, como variável dependente das doses de $\mathrm{P}\left(\mathrm{mg} \mathrm{dm}^{-3}\right)$ aplicadas na "cova" (Pc) e no solo “externo" (Pe), das quantidades de P aplicadas ( $\mathrm{mg}$ ) em todo o solo do vaso (Pav) e no solo da parte superior do vaso (Pas), nos solos LVAd-VI e LVAm-LS

Equação

Solo LVAd-VI

$\hat{\mathrm{P}}$ rs $=-1,312+0,019014 * \mathrm{Pc}+0,090930 * * \mathrm{Pe}$

$\hat{\mathrm{P}} \mathrm{rs}=2,066+0,009022 \mathrm{Pav}+0,000014^{* *} \mathrm{Pav}^{2}$

$\hat{\mathrm{P}}$ rs $=0,574+0,021407^{* *} \mathrm{Pas}+0,000017^{\circ} \mathrm{Pas}^{2}$

Solo LVAm-LS

$\hat{\mathrm{P}}$ rs $=-0,061+0,031609^{* *} \mathrm{Pc}+0,174831^{* *} \mathrm{Pe}$

$\hat{\mathrm{P}}_{\mathrm{rs}}=3,168+0,017720^{\circ} \mathrm{Pav}+0,000043^{*} \mathrm{Pav}^{2}$

$\hat{\mathrm{P}}_{\mathrm{rs}}=-0,042+0,061441^{* *} \mathrm{Pas}$
$\mathbf{R}^{2}$

0,981

0,938

0,986

0,972

0,952

0,969

${ }^{\circ}, *, * *$ : Significativos a 10,5 e $1 \%$, respectivamente.

Quadro 11. Equações de regressão da produção de matéria seca da parte aérea de plantas de milho (MS, em $\left.\mathrm{g}_{\text {vaso }}{ }^{-1}\right)$, como variável dependente do $\mathrm{P}$ recuperado $\left(\mathrm{mg} \mathrm{dm}^{-3}\right)$ pelo extrator Mehlich-1 da mistura do solo $\left(C+E_{1}\right)$ da parte superior do vaso (Prs), amostrada após a colheita do milho, nos solos LVAd-VI e LVAm-LS

\begin{tabular}{lcc}
\hline Solo & Equação & $\mathbf{R}^{2}$ \\
\hline LVAd-VI & $\hat{\mathrm{M} S}=-0,146+0,433038^{* *} \operatorname{Prs}$ & 0,641 \\
LVAm-LS & $\hat{\mathrm{M}} \mathrm{S}=0,320+0,315496^{* *} \operatorname{Prs}$ & 0,758 \\
\hline
\end{tabular}

**: Significativo a $1 \%$. 
A utilização de algum desses valores como um único nível crítico médio para o vaso não é confiável, pois o mesmo teor pode ser obtido a partir de diferentes combinações de doses de P na "cova" e no solo "externo".

\section{Simulação da amostragem do solo}

Quando se localiza o P, uma interrogação que ocorre ao amostrar o solo, para fins de calibração, diz respeito

Quadro 12. Níveis críticos médios de $\mathbf{P}$ no solo do vaso, após a colheita, para a obtenção de 90 \% da maior produção da parte aérea de plantas de milho, calculados a partir das equações ajustadas em função das doses de $P$ aplicadas na "cova" $e$ no solo "externo" (Pc e Pe), das quantidades de $P$ aplicadas em todo o solo do vaso (Pav) e na parte superior (Pas), nos solos LVAd-VI e LVAmLS

\begin{tabular}{lll}
\hline Solo & \multicolumn{3}{l}{ Variavél independente da equação utilizada } \\
\cline { 2 - 3 } & Pc e Pe Pav & Pas
\end{tabular}

\begin{tabular}{lccc}
\hline & \multicolumn{3}{c}{$\mathrm{mg} \mathrm{dm}^{-3} \mathrm{de} \mathrm{P}$} \\
\cline { 2 - 4 } LVAd-VI & 11,3 & 7,3 & 10,2 \\
LVAm-LS & 11,6 & 8,5 & 11,9 \\
\hline
\end{tabular}

à proporção de amostras a serem tomadas do sulco e das entrelinhas para compor uma amostra composta (Alvarez V. et al., 1986).

Foram feitas simulações para amostragem com coletas de 20 ou de 21 amostras simples. Para isso calculou-se, como único valor de $\mathrm{P}$ disponível para cada vaso, a média ponderada com o uso de diferente número de amostras simples e dos teores de $\mathrm{P}$ da "cova" e do solo "externo" (Pm), e ajustaram-se equações de regressão da produção de MS em função desses valores (Quadro 13).

Dessa forma, para o solo LVAd-VI, à medida que aumenta a proporção de amostras simples para a "cova", estreita-se a relação com a produção de MS, de modo que a melhor relação é obtida com toda a ponderação dada à "cova" (20 amostras). No solo LVAm-LS, o melhor ajuste (maior $\mathrm{R}^{2}$ ) foi obtido com a tomada de 7 amostras na "cova" e de 14 no solo "externo". Semelhante proporção foi utilizada para milho no campo (Alvarez V., 1982). No entanto, tal valor $\left(\mathrm{R}^{2}\right)$ é pouco superior aos obtidos com a tomada de 20 amostras na "cova".

Mesmo observando valores semelhantes de $\mathrm{R}^{2}$ para as equações de produção de MS em função do teor médio de $\mathrm{P}$, ponderado pelo número de amostras simples da "cova" e do solo "externo", deve-se atentar para o fato de que, no solo LVAd-VI, quando as doses variaram de $35+35(\mathrm{~T} 10)$ para $665+105 \mathrm{mg} \mathrm{dm}^{-3}$

Quadro 13. Equações de regressão da produção de matéria seca (MS) da parte aérea de plantas de milho $\left(\mathrm{g} \mathrm{vaso}^{-1}\right)$, como variável dependente da média ponderada $(\mathrm{Pm})$, pelo número de amostras simples, dos teores de $\mathrm{P}\left(\mathrm{mg} \mathrm{dm}^{-3} \mathrm{de} \mathrm{P}\right)$ recuperados da "cova" (Prc) e do solo "externo" (Pre), nos solos LVAd-VI e LVAm-LS

\begin{tabular}{|c|c|c|c|}
\hline \multicolumn{2}{|c|}{ Número(1) } & \multirow{2}{*}{ Equação } & \multirow{2}{*}{$\mathbf{R}^{2}$} \\
\hline Prc & Pre & & \\
\hline \multicolumn{4}{|c|}{ Solo LVAd-VI } \\
\hline 0 & 20 & $\hat{\mathrm{M}} \mathrm{S}=1,066+0,97725^{* *} \mathrm{Pm}-0,04483^{* *} \mathrm{Pm}^{2}$ & 0,154 \\
\hline 1 & 20 & $\hat{\mathrm{M}} \mathrm{S}=0,043+0,70279^{* *} \mathrm{Pm}-0,01741^{* *} \mathrm{Pm}^{2}$ & 0,322 \\
\hline 7 & 14 & $\hat{\mathrm{M}} \mathrm{S}=3,202-1,67258^{* *} \mathrm{Pm}^{1 / 2}+0,39759^{* *} \mathrm{Pm}$ & 0,944 \\
\hline 10 & 10 & $\hat{\mathrm{M}} \mathrm{S}=3,362-1,42392^{* *} \mathrm{Pm}^{1 / 2}+0,28762^{* *} \mathrm{Pm}$ & 0,970 \\
\hline 20 & 1 & $\hat{\mathrm{M}} \mathrm{S}=3,361-1,05110^{* *} \mathrm{Pm}^{1 / 2}+0,16271^{* *} \mathrm{Pm}$ & 0,983 \\
\hline 20 & 0 & $\hat{\mathrm{M}} \mathrm{S}=3,302-1,01065^{* *} \mathrm{Pm}^{1 / 2}+0,15456^{* *} \mathrm{Pm}$ & 0,983 \\
\hline \multicolumn{4}{|c|}{ Solo LVAm-LS } \\
\hline 0 & 20 & $\hat{\mathrm{M}} \mathrm{S}=1,243+0,65346^{* *} \mathrm{Pm}+0,0244^{* *} \mathrm{Pm}^{2}$ & 0,251 \\
\hline 1 & 20 & $\hat{\mathrm{M}} \mathrm{S}=1,063+0,31945^{* *} \mathrm{Pm}$ & 0,543 \\
\hline 7 & 14 & $\hat{\mathrm{M}} \mathrm{S}=0,715+0,15593^{* *} \mathrm{Pm}$ & 0,970 \\
\hline 10 & 10 & $\hat{\mathrm{M}} \mathrm{S}=1,576+0,05647^{* *} \mathrm{Pm}+0,00076^{*} \mathrm{Pm}^{2}$ & 0,966 \\
\hline 20 & 1 & $\hat{\mathrm{M}} \mathrm{S}=2,050+0,01540 \mathrm{Pm}+0,00038^{* *} \mathrm{Pm}^{2}$ & 0,950 \\
\hline 20 & 0 & $\hat{\mathrm{M}} \mathrm{S}=3,383-0,73575^{* *} \mathrm{Pm}^{1 / 2}+0,11015^{* *} \mathrm{Pm}$ & 0,949 \\
\hline
\end{tabular}

(1) Número de vezes que o $\mathrm{P}$ da "cova" ou solo "externo" é considerado no cálculo do teor médio ponderado de $\mathrm{P}$ (Pm); $*$, **: Significativos a 5 e $1 \%$, respectivamente. 
(T12) para a "cova" e para o solo "externo", o teor de Pm variou de 3,74 a 50,8 $\mathrm{mg} \mathrm{dm}^{-3}$, quando o número de amostras simples da "cova" e do solo "externo" foi 7 e 14, respectivamente, variou de 3,7 a $70,7 \mathrm{mg} \mathrm{dm}^{-3}$ para a proporção 10 e 10 e de 3,5 a $130,6 \mathrm{mg} \mathrm{dm}^{-3}$ para a proporção de 20 e 0 amostras simples da "cova" e do solo "externo".

Estas variações indicam que os valores das declividades do teor de $\mathrm{P}$ recuperado, em função das doses de $\mathrm{P}$ adicionadas $(\Delta \mathrm{Prp} / \Delta \mathrm{Pad})$, aumentaram conforme se aumentou o número de amostras simples da "cova". Aumento que justifica as modificações dos valores dos níveis críticos dos quadros 8 e 12 .

Assim, em concordância com a proposição da utilização de um único valor de nível crítico, o da "cova", sugere-se que a amostragem para fins de calibração da adubação fosfatada, para a forma de localização utilizada, seja feita apenas na cova.

Esta forma de amostragem significa que se passaria a dar maior ênfase na fertilidade do solo onde cai a semente e não na fertilidade média do terreno e, também, que dificilmente irá encontrar-se solos ricos em P que não precisem de adubação com fosfatos solúveis na instalação de lavouras de milho.

Esta recomendação necessita ser verificada em experimentos de campo, em que: as doses de $\mathrm{P}\left(\mathrm{kg} \mathrm{ha}^{-1}\right.$ de $\mathrm{P}_{2} \mathrm{O}_{5}$ ) deverão ser aplicadas no sulco de plantio em forma bem localizada; a amostragem deverá ser realizada nos sulcos de plantio. Depois, deverá estimar a curva que relaciona o $\mathrm{P}$ disponível $\left(\mathrm{mg} \mathrm{dm}^{-3} \mathrm{de} \mathrm{P}\right)$ do solo com as doses do $\mathrm{P}$ adicionadas $\left(\mathrm{kg} \mathrm{ha}^{-1} \mathrm{de}_{2} \mathrm{O}_{5}\right)$; também, a curva de resposta da produção em função das doses de $\mathrm{P}\left(\mathrm{kg} \mathrm{ha}^{-1}\right.$ de $\left.\mathrm{P}_{2} \mathrm{O}_{5}\right)$ e determinar a dose recomendável de $\mathrm{P}$ para a máxima eficiência econômica (MEE) e o nível crítico (NiCri) de P para a forma de amostragem feita.

Na utilização da análise de solo, para recomendação de adubação das lavouras, o esquema proposto seria o seguinte:

Amostragem do solo, após o preparo mecânico do terreno, para determinação do $\mathrm{P}$ disponível. E determinação da dose a ser recomendada (DR), por meio da seguinte fórmula:

$$
\mathrm{DR}=(\mathrm{NiCri}-\mathrm{Pd}) / \Delta(\operatorname{Prp} / \mathrm{Pad})
$$

\section{CONCLUSÕES}

1. A localização da maior quantidade de $\mathrm{P}$ aplicada no solo da "cova" aumenta a eficiência de utilização do $\mathrm{P}$ adicionado.

2. A produção de matéria seca da parte aérea de plantas de milho apresenta relação muito baixa com a quantidade de $\mathrm{P}$ aplicada por vaso (Pav), mas depende estreitamente das doses aplicadas à "cova" $(\mathrm{Pc})$ e ao solo "externo" (Pe), mostrando o efeito da localização do $\mathrm{P}$.
3. As superfícies de resposta $(\mathrm{MS}=\mathrm{f}(\mathrm{Pc}, \mathrm{Pe}))$ mostram que o crescimento inicial das plantas depende, essencialmente, das doses de $\mathrm{P}$ adicionadas na "cova".

4. Os valores dos níveis críticos para a "cova" são muito elevados e para o solo "externo" são baixos. Como a fração do volume da "cova" é pequena (1/10), a quantidade recomendável de $\mathrm{P}$ para o vaso não é muito elevada.

5. O valor de $\mathrm{P}$ disponível na amostra de solo da parte superior, retirada após a colheita $\left(\mathrm{C}+\mathrm{E}_{1}\right)$, representa bem a disponibilidade média do volume de solo amostrado em função do P adicionado na "cova" e na parte "externa". Porém, não se relaciona bem com a produção de matéria seca das plantas de milho.

6. Os níveis críticos médios de $\mathrm{P}$ no solo do vaso, após a colheita, são valores baixos e compatíveis com os obtidos usualmente em condições de campo.

7. A estreita relação entre a produção de matéria seca e o P recuperado da "cova" evidencia o uso de um valor de nível crítico para esse local. Assim, na fase de calibração, bastaria apenas a coleta de amostras na "cova" (sulco).

\section{LITERATURA CITADA}

ALVAREZ V., V.H. Efecto de los factores cantidad, intensidad y capacidad amortiguadora de fosfato en la evaluación del fósforo de suelos derivados de cenizas volcánicas de la Meseta Tarasca, Edo. Michoacán. Chapingo, México, Colegio de Postgraduados, 1982. 300p. (Tese de Doutorado)

ALVAREZ V., V.H. Correlação e calibração de métodos de análise de solos. In: ALVAREZ V., V.H.; FONTES, L.E.F. \& FONTES, M.P.F., eds. O solo nos grandes domínios morfoclimáticos do Brasil e o desenvolvimento sustentado. Viçosa, MG, UFV/DPS, SBCS, 1996. p.615-646.

ALVAREZ V., V.H.; CAJUSTE, L.J. \& CRUZ DIAZ, J. Estudio de la variabilidad en el contenido de fósforo del suelo en función de las dosis y ubicación del fertilizante fosfatado en un experimento de maiz (Zea mays L.). Agrociencia, 64:23-32, 1986.

ALVAREZ V., V.H. \& FONSECA, D.M. Definição de doses de fósforo para determinação da capacidade máxima de adsorção de fosfatos e para ensaios em casa-de-vegetação. R. Bras. Ci. Solo, 14:49-55, 1990.

ALVAREZ V., V.H.; NOVAIS, R.F.; DIAS, L.E. \& OLIVEIRA, J.A. Determinação e uso do fósforo remanescente, B. Inf., SBCS, 52:27-32, 2000.

ALVES, V.M.C.; MAGALHÃES, J.V.; VASCONCELLOS, C.A.; NOVAIS, R.F.; BAHIA FILHO, A.F.C.; FRANÇA, G.E.; OLIVEIRA, C.A. \& FRANÇA, C.C.M. Acúmulo de nitrogênio e fósforo em plantas de milho afetadas pelo suprimento parcial de fósforo às raízes. R. Bras. Ci. Solo, 23:299-305, 1999. 
ANGHINONI, I. Uso de fósforo pelo milho afetado pela fração de solo fertilizada com fosfato solúvel. R. Bras. Ci. Solo, 16:349-353, 1992.

BRAY, R.H. A nutrient mobility concept of soil plant relationships. Soil Sci., 78:9-22, 1954.

GENTA, H.L. \& CARNELLI, J. Muestreo de suelo para análisis de $\mathrm{P}$ en cultivo de caña de azúcar. Invest. Agron., 4:68-72, 1983.

GUARÇONI M., A. Efeito da localização de adubos fosfatados sobre o crescimento de plantas de milho. Viçosa, MG, Universidade Federal de Viçosa, 2001. 52p (Tese de Mestrado)

HOLFORD, I.C.R. Soil phosphorus: its measurement, and its uptake by plants. Aust. J. Soil Res., 35:227-239, 1997.

JUNGK, A. \& BARBER, S.A. Phosphate uptake rate of corn roots as related to proportion of the roots exposed to phosphate. Agron. J., 66:554-557, 1974.

KLEPKER, D. \& ANGHINONI, I. Crescimento radicular e aéreo do milho em vasos em função do nível de fósforo no solo e da localização do adubo fosfatado. R. Bras. Ci. Solo, 19:403-408, 1995.

LEITE, R.A. Uso de matrizes experimentais e de modelos estatísticos no estudo do equilíbrio fósforo-enxofre na cultura de soja em amostras de dois Latossolos de Minas Gerais. Viçosa, MG, Universidade Federal de Viçosa, 1984, p.87 (Tese de Mestrado)

MODEL, N.S. \& ANGHINONI, I. Resposta do milho a modos de aplicação de adubos e técnicas de preparo do solo. R. Bras. Ci. Solo, 16:55-59, 1992.

NEVES, J.C.L. Aspectos nutricionais em mudas de Eucalyptus spp - Tolerância ao alumínio e níveis críticos de fósforo no solo. Viçosa, MG, Universidade Federal de Viçosa, 1983. 87p. (Tese de Mestrado)

NICOLODI, M.; ANGHINONI, I. \& SALET, R.L. Alternativa à coleta de uma secção transversal, com pá de corte, na largura da entrelinha, na amostragem do solo em lavouras com adubação em linha no sistema plantio direto. R. Plantio Direto, 69:22-28, 2002.
NOVAIS, R.F.; FERREIRA, R.P.; NEVES, J.C.L. \& BARROS, N.F. Absorção de P e crescimento do milho com sistema radicular parcialmente exposto a fonte de fósforo. Pesq. Agropec. Bras., 20:749-754, 1985.

NOVAIS, R.F. \& SMYTH, T.J. Fósforo em solo e planta em condições tropicais. Viçosa, MG, Universidade Federal de Viçosa, 1999. 399p.

RANDALL, G.W. \& HOEFT, R.G. Placement methods for improved efficiency of $\mathrm{P}$ and $\mathrm{K}$ fertilizers: A review. J. Prod. Agric., 1:70-79, 1988.

ROMER, W. \& SCHILLING, G. Phosphorus requeriments of the wheat plant in various stages of its life cycle. Plant Soil, 91:221-229, 1986.

SCHLINDWEIN, J.A. \& ANGHINONI, I. Variabilidade dos índices de fertilidade do solo no sistema de plantio direto e coletas de amostras representativas. In: REUNIÃO SULBRASILEIRA DE CIÊNCIA DO SOLO, 2., Santa Maria, 1998. Resumos Expandidos. Santa Maria, Sociedade Brasileira de Ciência do Solo, 1998. p.142-145.

SLEIGHT, D.M.; SANDER, D.M. \& PETERSON, S.A. Effect of fertilizer phosphorus placement on the availability of phosphorus. Soil Sci. Soc. Am. J., 48:336-340, 1984.

STRIKER, R.B.; GILLIAN, J.W. \& JACKSON, W.A. Non uniform phosphorus distribution in the root zone of corn: growth and phosphorus uptake. Soil Sci. Soc. Am. Proc., 38:334-340, 1974.

VASCONCELOS, C.A.; SANTOS, H.L.; BAHIA FILHO, A.F.C.; OLIVEIRA, A.C. \& PACHECO, E.B. Amostragem de solo em área com adubação fosfatada aplicada a lanço e no sulco de plantio. R. Bras. Ci. Solo, 6:221-225, 1982.

WELCH, L.F.; MULVANEY, D.L.; BOONE, L.V.; McKIBBEN, G.E. \& PENDLETON, J.W. Relative efficiency of broadcast versus banded phosphorus for corn. Agron. J., 58:283-287, 1966.

ZHANG, J. \& BARBER, S.A. Maize root distribution between phosphorus - fertilized and unfertilized soil. Soil Sci. Soc. Am. J., 56:819-822, 1992. 